\title{
Estudio de la rigidez cadavérica que presenta la Síndone de Turín
}

\section{Study of the cadaveric rigidity presenting in the Shroud of Turin}

\section{Resumen}

En el estudio médico forense de la imagen sindónica llama la atención la intensa rigidez que se aprecia en la imagen corporal del Hombre de la Síndone. En este trabajo se estudia este fenómeno cadavérico y los posibles factores condicionantes que han podido modificar su normal evolución. Por otro lado, aprovechando la circunstancia de que esta rigidez se rompió y recuperó en hombros y brazos, se trata de determinar cronológicamente en que momento de la muerte se pudo producir tan sorprendente imagen.

Palabras clave: Rigidez cadavérica. Síndone de Turín. Tanatocronología.

\section{Abstract}

In the forensic study of the sindonic image it is necessary to pay attention to the intense rigidity that is observed in the corporal image of the Man of the Shroud. In this paper, this cadaveric phenomenon is analyzed and the possible conditioning factors which could have modified its normal evolution. Furthermore, taking into account the circumstance that this rigidity was broken and then recovered in shoulders and arms, we try to determine chronologically in which moment of the death this surprising image could be produced.

Key words: Cadaveric rigidity, Shroud of Turin, Time after death.

\section{JD. Villalaín}

\section{Catedrático de} Medicina Legal. Vicepresidente del Centro Español de Sindonología.

Correspondencia: Prof. Dr. José D. Villalaín. E-mail:

jose.d.villalain@uv.es

\section{Introducción}

Regularmente aparecen artículos y comunicaciones diversas que estudian diferentes aspectos de las sorprendentes imágenes que se contemplan en la Síndone de Turín. Como es sabido se trata de una gran sábana en la que aparece la imagen anterior y posterior de un hombre torturado. Según la tradición secular se trataría de la mortaja en que fue envuelto el cuerpo de Cristo después de muerto.

Buena parte de estas aportaciones son periodísticas, presentadas como tarea divulgativa, generalmente desarrolladas con la mejor intención aunque escasas de contenido científico; algunas buscando exponer diversas tesis apriorísticas, cargadas fantasía, de esoterismo o de ideología; las menos, analizando el lienzo y la imagen objetivamente, de acuerdo con los conocimientos científicos actuales.

Independientemente de su origen y del mecanismo de producción de la imagen sindónica, se trata de un lienzo funerario que refleja una muerte traumática y, por lo tanto, reúne todas las condiciones propias de la prueba y de los indicios biológicos del delito, por lo que debería analizarse con criterios medicolegales y médico forenses.

En una primera impresión llama la atención la intensa contracción, rigidez o tetanización que muestran estas imágenes. Dado que la rigidez se instaura progresivamente, el estudio de ésta, nivel alcanzado, características y evolución, pueden ofrecernos elementos que permitan determinar las circunstancias dinámicas propias de la impronta sindónica.
Fecha de recepción: 17-FEB-2010

Fecha de aceptación: 1-MAR-2010 
Las investigaciones científicas, cada vez más abundantes y de mejor nivel, que se realizan actualmente tanto sobre la Síndone de Turín como sobre el Sudario de Oviedo hacen que cada vez sea más probable que estas telas hayan estado en contacto con el cadáver de Jesús. Éste es el valor religioso principal de estas reliquias, pero desde el punto de vista medico legal, al tratarse de un sujeto, probablemente muerto por crucifixión, refleja un caso poco corriente que se ofrece excepcionalmente y que nos permite reflexionar sobre los conocimientos tanatológicos actuales.

Partiendo de esta realidad procederemos al estudio de la presunta rigidez que presenta el Hombre de la Síndone, utilizando para completarla y complementarla no sólo los datos que la tradición y los Evangelios nos han transmitido sobre la muerte de Jesús, sino también los datos proporcionados por el equipo EDICES, al que me honro en pertenecer, dedicado a la investigación y estudio del Sudario de Oviedo y los conocimientos diversos que este estudio proporciona.

\section{¿Estaba rígido el Hombre de la Síndone?}

No suele tratarse en los libros y monografías sobre la Síndone, el tema de la rigidez cadavérica, sino de pasada y de un modo general. Ni siquiera en los de carácter médico. Pero tácita o implícitamente, todos los autores en los trabajos y análisis sindónicos, o simplemente sobre la imagen de la Sábana están de acuerdo en la existencia de una intensa rigidez en el Hombre de la Síndone. Todos los autores, al describir la imagen sindónica, sin referirse a una rigidez cadavérica, la describen como musculada.

Por lo general, los autores se limitan a afirmar que el Hombre de la Síndone presentaba "rigor mortis", como hace, por ejemplo, Novelli, o señalan que se encontraba en rigidez cadavérica (Stevenson, Habermans, Jackson et al., y tantos otros.); uno de mis maestros: Royo Villanova señala concretamente que "el estudio medicolegal de la Sábana Santa de Turín... ha comprobado el "rigor mortis" del cuerpo inerte del Redentor".

En los primeros estudios médicos que se hicieron sobre la Síndone, los autores hablan de tetanización. Le Bec refiere un estado de tetanización de todo el cuerpo. Caminals, escribe que el Hombre de la Síndone tenía el pecho levantado por tetania asfixiante y epigastrio retraído. La misma expresión es la que usa Hynek y escribe este autor: "La excesiva rigidez de los músculos, tal como se presenta en las fotos de Enrie, me recordó un hecho que luego he venido comprobando constantemente: a consecuencia de la forzada tensión impuesta a los músculos, la rigidez mortal se manifiesta inmediatamente después del ultimo suspiro". En otro de sus libros se limita a decir que la imagen tiene "la típica rigidez mortal", aunque en otro de ellos analiza la rigidez con detenimiento y la compara con testimonios y experiencias propias. Siliato hace suya la frase de Antoine Legrand: "Después de la muerte, el cadáver quedó enseguida extremadamente rígido".

El célebre anatomopatólogo norteamericano, Robert Bucklin, añade "The body appears to be in state of rigor mortis which is evidenced by an overall stiffness as web as specific alterations in the appearence of the lower extremities from the posterior aspects. The imprint of the right calf is much more distinct than that of the left leg was rotated in such a way that the sole of the left foot rested on the ventral surface of the right foot with resultant slinght flexion of the left knee. That position was maintained after rigor mortis had developed" (El cuerpo parece estar en estado de rigor mortis lo que se evidencia por una rigidez general así como alteraciones específicas en la posición de las extremidades vistas desde el plano posterior. La huella de la pantorrilla derecha es mucho más precisa que la de la pierna izquierda la cual fue rotada de modo que la planta del pie izquierdo descansaba en la superficie ventral del pie derecho con resultado de una ligera flexión de la rodilla izquierda. Esta posición fue mantenida después de que el rigor mortis se hubiera desarrollado). y, en otro lugar: "que el cuerpo estaba definitiva y obviamente rígido y tenso, la pierna izquierda encogida en la posición que tenía durante la crucifixión, habiendo quedado fija así; debido a la rigidez cadavérica, la cabeza definitivamente inclinada hacia delante".

Los expertos en Medicina Legal subrayan "la acentuada rigidez del Hombre de la Síndone, característica de las muertes excepcionalmente fatigosas y dolorosas". Algunos autores añaden "con la cabeza hundida en el pecho, rasgo típico de los crucificados". Según Hynek "la rigidez cadavérica en los crucificados... era más pronunciada en las extremidades superiores" y lo achacaba al esfuerzo de suspensión.

Una serie de relatos complementarios en la que se cuentan situaciones dramáticas de muertes y tortura por colgamiento, dieron mucha luz al mecanismo de producción.

Cuenta Solé que en una conferencia de Antoine Legran, un espectador llamado R. Gieser, luxemburgués de nacionalidad, que había sido enrolado a la fuerza en el ejército alemán y deportado luego al campo de 
concentración de Dachau, expuso su experiencia en este campo de concentración donde contempló numerosas muertes por colgamiento. Posteriormente, P. G. Delory, sacerdote que había estado en ese campo, confirmó el relato, lo mismo que dos exprisioneros de Lager. En todos estos casos se refiere un copioso sudor y la fiebre del torturado y concluye: "El condenado moría con la cabeza hundida en el pecho, hasta tal punto metida en los omóplatos, que casi quedaba a su nivel"..."la cabeza caía hacia delante en el eje del cuerpo cuya rigidez era extrema". Estos testimonios se completan con los relatos aportados por Hynek que presenció, en la guerra de 1914-1918, el castigo por colgamiento, Ilamado aufbinden, que se aplicaba a los soldados austrohúngaros y que confirmaba aquella sintomatología. Estos cuadros se repiten en los interrogatorios policiales de numerosos paises en que la tortura no se mide correctamente y se sucede de la muerte del interrogado.

Ese mismo criterio lo mantiene el Prof. Valdés que se limita a decir que "el cuerpo del Señor entró rápidamente en la rigidez postmortal", postulado que recoge también J Loring. Mario Cappi señala muy agudamente que la fijación del cuerpo en la postura semiflexionada general demuestra que estaba muerto y rígido cuando se le quitó de la cruz. Por lo tanto, el rigor mortis se presentó en un momento muy precoz. Así lo afirma Barbet cuando señala que "cuando muere queda instantáneamente rígido. La rigidez debió ser brutal, instantánea, total, de un solo golpe". Para Hynek fue instantánea. La compara con la que se produce en los ciervos acosados y sometidos a una fatiga excesiva. Gedda, a la hora de calcular la estatura, tuvo en cuenta el estado de rigidez y escribía al respecto que "el tórax de Jesús estaba agitado, expandido, globuloso". Siliato añade, a propósito de la rigidez cadavérica, que es probable "que eso haya impedido a los sepultureros colocar el cadáver con los brazos a lo largo de las caderas, única postura posible (sobre el pubis), dada la repentina rigidez cadavérica del cuerpo".

Se trata, por tanto, según observaciones propias y el criterio unánime de una rigidez cadavérica, instantánea o muy precoz, que era completa cuando se procedió a descolgarlo, intensa y que hubo de ser vencida para recoger los brazos y proceder a amortajarlo.

Domínguez resume de modo completo la situación de rigidez en que se encuentra el Hombre de la Síndone y dice que el Hombre sindónico presenta una acentuada rigidez, propia, por otro lado, de las muertes en extremo fatigosas y dolorosas con deshidratación y fiebre.
Esta rigidez se caracteriza principalmente por:

- Cabeza flexionada hacia delante y nuca elevada y tensa.

- Músculos esternocleidomastoideos, trapecio e inspiradores rígidos, lo mismo que el deltoides.

- El tórax, se encuentra dilatado, en inspiración forzada.

- Los músculos pectorales mayores se ven contraídos y salientes.

- La musculatura escapular se encuentra contraída y pegada a las costillas.

- Epigastrio hundido e hipogastrio prominente.

- Musculatura dorso-lumbar tensa.

- Lordosis lumbar acentuada.

- Glúteos intensamente marcados, especialmente el derecho.

- Piernas flexionadas, especialmente la derecha.

- Pie izquierdo más hiperextendido que el derecho.

Añade Domínguez que los brazos, en cambio, no presentan rigidez equiparable a la del resto del cuerpo y fueron cruzados sobre el pubis sin un esfuerzo excesivo, al contrario que las piernas, criterio subjetivo pero no obstante valorable porque procede de un médico con amplia experiencia.

En síntesis: no parece que haya dudas de que la imagen de la Síndone reproduce un cuerpo en estado de intensa rigidez. Que esta rigidez se produjo, por la morfología que presenta el cuerpo, estando éste colgado. Que para colocar las manos del cadáver sobre la zona pubiana hubo que vencer esta rigidez. Que la rigidez volvió a implantarse en las manos, brazos y hombros después de haber sido vencida.

\section{¿Estaba muerto el Hombre de la Síndone?}

Pese a la forzada controversia que mantienen algunos medios de comunicación en la actualidad, sin duda hay que afirmar que sí. Los signos que proporciona la propia síndone y los relatos evangélicos son demostrativos. Intentar demostrar lo contrario no es sino un empecinamiento y un razonamiento tortuoso que no tiene base científica seria.

- Según el Sudario dicho sujeto era cadáver. El mecanismo de formación de las manchas es incompatible con cualquier posible movimiento 
respiratorio y, además, se ha demostrado que en la tela hay sangre postmortem.

- Según los Evangelios existen numerosos testigos, algunos conocedores por oficio de la muerte en cruz, otros interesados en la destrucción del Maestro. Presenciaron la muerte: Juan, los soldados, el centurión, José de Arimatea, los seguidores de Jesús presentes, las mujeres, sacerdotes, pontífices y fariseos, personalmente o representados.

- Según la Síndone. En la propia tela se ha demostrado que la herida del costado tenía carácter postmortem, la lanzada supone una grave herida en el corazón o a nivel pleuropulmonar; la facies hipocrática de la imagen es cadavérica; el cuerpo estaba en una situación de rigidez intensa a todos los niveles y en todas las regiones; el tórax estaba en inspiración; la misma posición de brazos y manos sobre pubis, inmóviles, tras vencer la rigidez, así nos lo indica; los miembros inferiores aparecen asimétricos con las piernas flexionadas, en una postura antigravitatoria mantenida; la cabeza flexionada sobre el tórax y éste sobre el abdomen, en una postura mantenida contra la gravedad; el vientre hacia fuera; se ha comprobado la existencia de sangre vital y postmortem y, sobre todo, la quietud absoluta que presenta la imagen es incompatible con cualquier hálito de vida. El mismo detallismo de la imagen es incompatible con cualquier movimiento por pequeño que fuese.

\section{Notas sobre la rigidez cadavérica}

Desde el punto de vista médico cabe distinguir dos tipos de contracción muscular, estructuralmente similares pero con distintas secuencias enzimáticas. En efecto, la contracción postmortem no tiene una función práctica determinada y sólo revela la consecución y agotamiento de unas reservas de energía del músculo tras la muerte celular.

\section{Introducción}

Al sobrevenir la muerte, se origina un estado de relajamiento o flacidez muscular generalizado; posterior- mente, lenta y progresivamente, se instaura un proceso de contracción muscular. La rigidez cadavérica, por tanto, sería el estado de tensión muscular progresiva temporal que sobreviene después del fallecimiento*.

Se acompaña de cambios en el patrón bioquímico muscular: producción de lactato, acidez muscular y pérdida de trifosfato de adenosina (ATP). Se caracteriza por un notable descenso de la extensibilidad muscular y pérdida de la recuperación de la deformidad de las fibras musculares.

\section{Evolución}

Todos los músculos entran en rigidez tanto si son de fibra lisa como de fibra estriada, según la doctrina general. Los órganos que tienen músculos de fibra lisa, el corazón y el diafragma están rígidos entre 30 minutos a 2 horas tras la muerte La musculatura estriada esquelética inicia el rigor de 3 a 6 horas, del fallecimiento.

La rigidez aparece progresivamente y tiene mucho que ver, según nuestra experiencia, con la posición del cadáver: En un cadáver en decúbito supino y con la cabeza levantada respecto al plano corporal, se produce en el orden siguiente, según la mayoría de los autores: mandíbula inferior y músculos orbiculares de los párpados, cara, cuello, brazos, piernas, tronco. En otras posturas, el orden de producción varía con la postura. El cadáver acaba constituyendo un bloque solidario, fijo por la rigidez cadavérica. Pero la secuencia con que se instaura el rigor mortis no es uniforme en los distintos autores: Según los esquemas de Nysten, la rigidez comienza por la mandíbula y la nuca, seguida del tronco, miembros superiores y miembros inferiores. Brouardel ha escrito que la rigidez cadavérica comienza por las vesículas seminales, probablemente pensando en los cuadros asfícticos, y sigue por la mandíbula, cuello y resto de los músculos. Barahona afirma que empieza por el maxilar inferior, enseguida el cuello y párpados, luego miembros inferiores y, por último, los músculos de los superiores y del tronco. Según Sommer-Larcher, se inicia por la mandíbula, casi simultáneamente miembros inferiores y nuca; después los miembros superiores. Brouardel afirma que la rigidez de los miembros inferiores es previa a la de los superiores, que los músculos de los dedos son los que mantienen la rigidez más tiempo y que el órgano que ha sido el último en entrar en rigidez, es también

\footnotetext{
"Louis, uno de los pioneros de la Medicina Legal, en su cuarta carta escribe: "Por investigaciones hechas con toda la exactitud que me ha sido posible, y que he continuado por espacio de muchos años sin interrupción, he visto más de quinientos individuos que en el instante de la muerte, es decir, en el momento de la cesación absoluta de los movimientos del cuerpo, las articulaciones empiezan a ponerse rígidas, aun antes de la disminución del calor natural".
} 
el último en perderla. Esto es debido a la variabilidad que ofrece, a la experiencia de los autores y al desconocimiento que existía en los más antiguos sobre la fisicoquímica del fenómeno.

En cualquier caso se instaura una contractura atlética isométrica, resaltando los relieves musculares, con un ligero predominio flexor. Por el contrario, teniendo en cuenta la importancia que tiene el grado de hidratación o deshidratación muscular si el cadáver quedase en posición vertical invertida, con los pies más elevados, el ritmo se hace inverso. En el caso de un crucificado la deshidratación corporal superior y el edema y congestión inferior hacen que la rigidez comience muy rápidamente por arriba y sea lenta en la parte inferior.

En condiciones normales, personas bien alimentadas, con su sistema nervioso y muscular intactos y muertes naturales, la rigidez comienza en la articulación temporomandibular entre 2 y 4 horas después de la muerte. Posteriormente se extiende a toda la musculatura siendo completa a las 8-12 horas y es máxima a las 24 horas. Inicia su desaparición a las 36-48 horas y termina a los 2 a 4 días de la muerte. Se ha generalizado la Ilamada regla de $\mathrm{Ni}$ derkorn, según la cual, la rigidez es precoz antes de 3 horas; normal entre 3 y 6 horas; tardía entre las 6 y 9 horas y muy tardía cuando pasa de este plazo.

Mallach publicó en 1964 un impresionante estudio. Según este autor, la rigidez empieza entre 2 y 8 horas, con una desviación estándar superior a 5 . El período previo oscila entre menos de media hora a 7 , con una desviación estándar de $3 \pm 2$. Estas cifras se determinaron valorando 26 publicaciones comprendidas entre los años 1811 y 1960. La rigidez completa osciló entre 6 y 10 horas con variaciones entre 2 y 20 , evaluando 28 publicaciones del mismo período con una desviación estándar de $8 \pm 1$ sobre el tema. Son cifras medias aproximadas. Ya Schleyer ha señalado el "considerable dogmatismo" con que tratan el tema los libros. Shapiro, Lyel, Stemmer, Cleveland y otros autores han señalado que la variabilidad en las cifras que se consideran es debida a que la rigidez cadavérica es la expresión de un proceso fisicoquímico y los datos disponibles tienen muy diversos orígenes: unos proceden de cuerpos tendidos en su cama; otros sobre una mesa de autopsia y los que se examinan han muerto en situaciones extremas, externas o internas, cadáveres de personas lesionadas y los que se obtienen experimentalmente proceden de pequeñas masas musculares y pequeños animales de laboratorio que no son comparables a un cuerpo humano completo.
Tratándose, en el caso de la imagen sindónica, de una rigidez aparentemente temprana veamos lo que recoge la bibliografía al respecto. En efecto, BrownSequard publicó el caso de un soldado que murió por una fiebre tifoidea complicada con un profundo agotamiento. En este caso la rigidez precedió tres minutos a la parada cardiaca. El mismo autor describió cómo en los casos de cólera que cursan con calambres, se declara una rigidez precoz en los músculos afectos. Bonnet afirma que la rigidez se inicia después (¿inmediatamente?) de ocurrida la muerte, alcanza su totalidad a las quince horas y luego desaparece lentamente. Nysten ha llamado la atención sobre cómo en los casos de marasmo y caquexia general por tuberculosis pulmonar o cáncer de estómago, la rigidez es también muy precoz, escasa y corta. Sommer fijaba el comienzo entre 10 minutos a siete horas tras la muerte. Peiró refiere este plazo comprendido entre 10 minutos y 16 ó 18 horas. Según las observaciones del Instituto Oscar Freire de Sao Paulo, que tiene una experiencia inmensa, la rigidez cadavérica aparece en la primera hora después de la muerte; Maschka nunca la vio antes de hora y media; Hofmann o Niderkorn lo marcan en dos horas, siendo completa antes de tres horas; Sydney Smith en tres horas o poco antes; Borri, Strassmann o Balthazard, tres horas. Por lo tanto, en la literatura se describen casos precoces, semejantes al que presenta el Hombre de la Síndone. La rapidez de aparición y la cortedad de la rigidez es también conocida en los casos de animales forzados o fatigados en la carrera, cinco minutos después de la muerte. Vock y cols., han publicado un caso de rigidez instantánea en un caso de tetania. Se han descrito casos de rigidez instantánea sobre todo en cuadros que cursan con convulsiones, por ejemplo intoxicaciones por estricnina o insecticidas organofosforados; Morgagni ha citado casos de muerte repentina en que la rigidez se presentó casi inmediatamente; Sommer, Muller y otros muchos han visto que la rigidez se presenta más precozmente después de enfermedades agudas y caquectizantes, a veces de modo casi instantáneo. Balthazard refiere que la rigidez puede aparecer inmediatamente después de la muerte en los sujetos que han sucumbido al tétanos o al envenenamiento por estricnina. Browwn-Sequard en sujetos fallecidos debido a enfermedades infecciosas.

\section{Mecanismo de producción}

Actualmente no se conoce de modo absoluto y con precisión el mecanismo, sin embargo existen numerosos datos comprobados y aplicables fruto de la investigación de estos últimos años. Se han propuesto varias teorías bioquímicas todas ellas muy complejas y solo demostradas en parte, pero, sintetizando en lo posible, puede afirmarse que el ATP (adenosintrifosfórico) 
se transforma en ADP (adenosindifosfórico), liberando una molécula de ácido fosfórico. Este último proporciona el fosfato necesario para la resíntesis del ATP, utilizando como dador de energía el glucógeno. Cuando la reserva de glucógeno se ha agotado, falta energía para resintetizar ATP y éste se transforma en ADP definitivamente, originando la contracción de la miosina. Por lo tanto la reserva de glucógeno es fundamental y en el caso de un sujeto que ha muerto como el Hombre sindónico ésta tenía, necesariamente, que ser mínima.

\section{Desarrollo}

Casper esquematiza la evolución del rigor mortis, de modo parecido a como lo hace Camps a tenor del siguiente esquema, aplicable a la práctica médico forense:

- Si el cuerpo está caliente y relajado, la muerte se ha producido menos de 3 horas antes.

- Si el cuerpo esta caliente y parcialmente rígido, la muerte se ha producido entre 3 y 8 horas antes ( 2 a 9 horas en el esquema de Camps).

- Si el cuerpo está frío y rígido, el intervalo postmortem es de 8 a 36 horas (más de 9 horas según Camps).

- Si el cuerpo está frío y flácido, el intervalo es mayor de 36 horas.

Niederkorn, tras estudiar la evolución de la rigidez cadavérica en 113 cadáveres, obtuvo los siguientes resultados: En 2 casos fue completa a las 2 horas; en 76 casos, la rigidez era completa entre 4 y 7 horas; en 31 casos, después de 4 horas; en 14 casos, se produjo después de 5 horas; en 20 casos, después de 6 y en 11 casos, tras 7 horas; en otros 2, tras 13 horas de evolución.

Bendall distinguió en la marcha del rigor mortis tres fases: fase de retraso o pre-rigor, fase rápida y fase de post-rigor, fase esta última que no tiene interés a nuestros efectos porque estudiamos una rigidez establecida.

La fase de retraso mantiene las propiedades musculares. Se caracteriza porque las cifras de ATP permanecen invariables, mientras que decrecen rápidamente los valores de fosfocreatinina y de $\mathrm{pH}$. El ATP se transforma en ADP y éste, junto con el fósforo y los hidrogeniones, reacciona con la glucosa liberada de los depósitos de glucógeno. En esta fase una tercera parte del ADP se reconvierte por medio de la creatinquinasa utilizando fosfocreatinina. Por lo tanto la reserva de glucógeno es fundamental en el proceso.
La fase rápida está caracterizada por la pérdida de la extensibilidad y comienzo de los acortamientos marcados en la fibra muscular. Se inicia la pérdida de ATP y la concomitante formación de amoníaco debido a la defosforilización y deaminación de los adenin nucleótidos. Aparece inopina trifosfato (ITP) e inopina difosfato (IDP) que alcanza el máximo al final de la fase.

Por lo tanto, desde nuestro particular punto de vista, en relación a la instauración del rigor mortis, la cantidad de glucógeno es inversamente proporcional al tiempo que se necesita para iniciarla. Es este un factor, que ya hemos señalado, muy importante en la muerte de Jesús.

Por otro lado, las reservas de glucógeno están relacionadas con el $\mathrm{pH}$; valores bajos de $\mathrm{pH}(5,6)$ reflejan cifras elevadas de glucógeno; cifras altas $(7,2)$ indican reservas bajas o nulas. El contenido de lactato corre paralelo a las cifras de glucógeno, por eso las cifras de glucógeno se refieren en unidades de lactato, de tal modo que una unidad de lactato equivale a media unidad de glucosa derivada del glucógeno.

En todo el mecanismo tiene también una importante función el calcio, como desencadenante del rigor y mecanismo patogénico de su iniciación. En nuestro caso las cifras de calcio debían ser correctas y más bien altas en un sujeto acostumbrado a la vida al aire libre, sin signos de raquitismo o deficiencias cálcicas.

\section{Duración}

Nysten definió la "Ley de la rigidez cadavérica" determinando que la intensidad y duración están relacionadas, de modo que cuando la rigidez es precoz, ésta es escasa y breve, mientras que si es tardía, es notable y prolongada. Hay que tener en cuenta que existen procesos que no siguen estas reglas (electrocución, muertes por frío, cansancio). Acelera su presentación cuando la actividad metabólica es grande en el momento de morir, en los estados febriles, en los esfuerzos o en verano.

La duración del rigor varía mucho en la práctica y depende, en opinión de Bendall, de la cantidad inicial de fosfocreatinina y de los depósitos de glucógeno, condicionado todo por la temperatura. A temperatura constante de $37^{\circ}$ el rigor puede variar desde media hora en un sujeto exhausto hasta cuatro o más horas en sujeto descansado y bien alimentado. Es un fenómeno generalizado en todos los animales (conejo, buey, cordero, hombre) pero no pueden olvidarse excepciones como las ranas y 
ballenas que presentan grandes retrasos o el pollo en que se inicia muy rápidamente. Por lo tanto en los datos experimentales sobre animales es muy importante conocer y valorar la especie antes de hacer traspolaciones rápidas.

\section{Reinstauración}

Gisbert señala, como todos los autores modernos, que en la fase de instauración "la rigidez puede vencerse aplicando cierta fuerza, recuperando los miembros su flacidez, pero al cabo de un cierto tiempo se reinicia el proceso, volviendo de nuevo los músculos a ponerse rígidos". Según Thoinot, y como norma general, puede afirmarse que, en condiciones normales, si en las primeras 7-8 horas vencemos la rigidez aplicando una fuerza externa, se restaura espontáneamente, aunque de forma menos manifiesta, en un período de cuatro a seis horas. Pasadas 8 ó 9 horas la rigidez no es recuperable. En el caso sindónico, la rigidez de los hombros se vence para colocar los miembros superiores sobre el abdomen.

También hay que tener en cuenta que en la fase de estado, la rigidez es de tal intensidad que puede originar desgarros el forzarla y, añade el propio Gisbert, que "en la segunda fase o período de estado, la rigidez es prácticamente invencible sin producir desgarros o fracturas". Hoy se sabe, y la experiencia lo confirma, que cuando la rigidez está establecida el esfuerzo que debe aplicarse es considerable, pudiendo originarse roturas fibrilares, luxaciones o malposiciones articulares, fenómeno repetitivo en las salas de autopsia cuando se vence la rigidez para iniciar la necropsia. Es éste un punto que debe considerarse también en el caso que estudiamos porque la subluxación del hombro, que presenta el Hombre de la Síndone, cuya explicación se ha intentado de tantas formas, tiene claramente este origen. La rigidez en este caso estaba plenamente establecida.

En la fase de resolución, si se vence la rigidez, ésta no se recupera. Decía Louis, hace ya muchos años, que si se fuerza una articulación rígida se vuelve indiferente para todos los movimientos, un criterio demasiado simplista, propio de un pionero de la Medicina Legal, porque otros muchos autores, especialmente Thoinot han puntualizado este extremo cuando afirman que el hecho referido es válido para el período de estado, puesto que las articulaciones si se fuerzan al comienzo del proceso se vuelven otra vez rígidas al cabo de unas horas. Niederkorn ha concluido en sus estudios que si se vence la rigidez en el período de estado, se vuelve a instaurar con una intensidad inversamente proporcional al período postmortal. A las 20 horas el fenómeno ya no se produce.

\section{Desarrollo y circunstancias modificadoras}

Rodríguez Pazos ha llamado la atención sobre el escaso número de investigaciones realizadas en músculo esquelético humano. La mayor parte de las investigaciones sobre muerte celular muscular han sido hechas en miocardio y pocas sobre músculo esquelético y estas investigaciones en cadáveres animales, de ahí las opiniones existentes que dependen muchas veces, más del subjetivismo y la experiencia de los autores que de mediciones experimentales rigurosas.

Existen también numerosos factores, que se han ido recogiendo en la literatura medicolegal a través de los años, que condicionan la aparición de la rigidez, según el cuadro general expuesto. Por lo tanto, al objeto de calcular cómo y cuándo se produce la rigidez del cadáver, es necesario tener en cuenta tanto las circunstancias extrínsecas o externas como las propias del sujeto.

Desde este punto de vista se hace necesario que analicemos la muerte de Jesucristo a través de los datos conocidos, que se corroboran en la Síndone de Turín y en el Sudario de Oviedo, para, a la vista de éstos, analizarlos como factores modificadores.

La muerte en cruz es una muerte fundamentalmente asfíctica. En la imagen sindónica se reflejan dos posturas reiteradas originadas por el "hambre de aire" que experimentaba el reo. Esta situación suponía un enorme ejercicio muscular, fatiga creciente, acumulación de metabolitos musculares, dolor muscular y calambres reiterados, linfocitosis y luego neutrofilia (35.000 leucocitos), hipersecreción de las glándulas suprarrenales que origina aumento de la tensión arterial compensatoria, una disminución de los eosinófilos y aumento de la actividad fibrinolítica.

Los sujetos muertos por colgamiento tienen una fiebre intensa del orden de $40-41^{\circ} \mathrm{C}$ y más, hipoglucemia con debilidad acentuada, sudoración profusa, visión borrosa, cefalea, palpitaciones, náuseas incluso vómitos, deshidratación intensa debido a la falta de reposición de líquidos, ejercicio, pérdida por la respiración y las hemorragias. Consecuentemente, intensa sed.

Como consecuencia de la desviación de líquidos al espacio intratisular y de la desviación de sangre a periferia y a los planos inferiores, se acentúa la deshidratación general y, sobre todo, de la mitad corporal superior.

Sobre este cuadro asfíctico general se fue imponiendo el shock que, en el caso de Jesús fue múltiple: En primer lugar un shock psicógeno que experimentó 
en el Huerto de los Olivos, que originó pérdida de agua y de sangre y que ablandó la piel ante futuras lesiones. En segundo lugar se originó un shock neurogénico debido al dolor continuo que experimentó durante toda la Pasión (corona de espinas, golpes, maltrato, flagelación y lesión del nervio mediano), potenciado por el indudable componente emocional que originaba el conocimiento y su lógica sensibilidad.

En tercer lugar, un shock hipovolémico originado por las pérdidas sanguíneas debidas a la hematidrosis, hemorragias de la flagelación y la crucifixión con una intensa sed que aumentaba la angustia y el dolor. El shock hipovolémico sería la causa fundamental porque origina daño hipóxico a nivel de microcirculación, secuestro e hipóstasis sanguínea, aumento de la permeabilidad parietal vascular, acidosis tisular, cierre de circuitos vasculares, reducción de volemia, del retorno venoso y del volumen cardíaco, disminución del volumen plasmático, aumento de la agregabilidad plaquetaria, liberación de activadores plasmáticos, aumento de vasopresina y angiotensina, pulmón de shock con profunda alteración de la relación ventilación/perfusión y edema pulmonar que se potencia por el componente distributivo.

En cuarto lugar, un shock distributivo originado por la asfixia y por la posición anómala colgado que supone una inmovilidad vertical, que origina una caída progresiva de la tensión arterial, como se aprecia en la imagen anómala péndula abdominal.

Por último, un shock cardiogénico que origina alteraciones metabólicas cardíacas y la detención final del corazón.

Este criterio viene ratificado por el Sudario de Oviedo, según el cual, el sujeto murió colgado, a consecuencia de un fracaso cardíaco que originó un gran encharcamiento y edema pulmonar.

\section{Circunstancias intrínsecas}

Empíricamente, Brown-Sequard había comprobado que la rigidez depende del estado de conservación e integridad de la musculatura. Una muerte natural sin afectación nutricional, integridad muscular y sin violencia origina una rigidez intensa, tardía y duradera. En el Hombre de la Síndone las masas musculares están tremendamente contundidas y son numerosas las heridas contusas, que vienen a cubrir el $50 \%$ del cuerpo, según los cálculos de Diggio. Por lo tanto, el grado de integridad muscular era muy deficiente. Siguiendo las leyes de Nysten, en sujetos musculares e individuos con buena nutrición, la rigidez es tardía, intensa y duradera; a la inversa, la destrucción y las lesiones musculares hacen que la rigidez sea precoz, corta y débil. Por la misma razón en personas cansadas, agotadas, como es el caso de Jesús, la rigidez aparece cercana y débil (Brown-Sequard). Debe considerarse también la posible actividad muscular violenta previa, originada por traslados, maltratos y crisis asfícticas en la cruz. Husband ha estudiado alguno de los factores que modifican la rigidez, entre ellos el ejercicio violento previo a la muerte. En estos casos la rigidez desaparece rápidamente y es precoz, débil y corta. En todos ellos, la disponibilidad de glucógeno y adenosintrifosfatasa es menor y ambas sustancias son esenciales en la aparición de la rigidez. En este tipo de muerte la hipoglucemia es la regla al agotarse las reservas de glucógeno. Por eso, actividad muscular, acidez y fiebre acortan el proceso.

El sexo es un factor condicionante que debe tenerse en cuenta, no ya en el proceso de rigidez sino incluso morfométrica e histoquímicamente. Brooke y Engel han descrito estas diferencias que, junto a la edad, modifican la funcionalidad contractiva. En el hombre es más intensa, tardía y duradera que en la mujer, sin embargo el deterioro físico de Cristo hace que no pueda valorarse este apartado.

La edad influye igualmente. En niños y viejos es pre$\mathrm{coz}$, débil y corta. Así se ha descrito una rigidez prepuberal, puberal, juvenil y media donde la rigidez se hace intensa, tardía y duradera. En el caso que estudiamos, se trata de un sujeto en plenitud pero muy deteriorado por el maltrato, en consecuencia este factor tampoco es aplicable.

El tipo de muerte es otro elemento que aporta una gran variabilidad. La fiebre origina una rigidez precoz, débil y corta. En el caso de Jesucristo la fiebre al morir tuvo que ser extremadamente alta. Lo mismo ocurre cuando precede una agonía larga. En estos casos la rigidez también es precoz, débil y corta. Una agonía con convulsiones (tétanos, intoxicación estrícnica, intoxicación por alcaloides (Taylor), o insecticidas), originan una rigidez que es precoz, intensa y larga. Se conoce este efecto en otras muchas intoxicaciones como $\mathrm{CO}$, As, o Cloroformo que también la hacen precoz, intensa y larga, debido a las convulsiones que preceden a la muerte. La electrocución, que origina una muerte con fuerte contractura muscular o convulsiones, origina una rigidez precoz, intensa y larga, como describió el propio Lombroso.

\section{Factores de origen externo}

La temperatura exterior, al igual que sucedía con la interior, es muy importante en la instauración y, sobre todo, en la evolución tanatocronológica. Como 
la rigidez es consecuencia de un proceso bioquímico, influye enormemente la temperatura; por regla general el calor exterior hace la rigidez precoz e intensa y su conclusión también rápida.

Nysten ya había puesto en evidencia que el rigor mortis, aumenta con la temperatura; Kussmaul, Husband, Morgenstern, Foster y cols., Bendall y Davey, así lo han registrado entre otros muchos. Tanto el calor exterior como el interior (sobre todo la fiebre) hacen la rigidez precoz e intensa y su conclusión también rápida. En las series experimentales realizadas por Brier y Freund, temperaturas altas ambientales han producido rigideces muy precoces; a partir de $50^{\circ} \mathrm{C}$ el tiempo oscila entre instantáneo y 15 minutos, alcanzándose la rigidez plena entre 10 y 45 minutos. En contra, es admitido por todos los autores, que el frío hace la rigidez tardía y prolongada. Por debajo de $10^{\circ} \mathrm{C}$ no suele observarse.

Hoy se sabe que la rigidez cadavérica se desarrolla en tres fases bien definidas (ya expuestas con detalle antes):

- Fase de retraso que mantiene las propiedades musculares.

- Fase rápida caracterizada por la pérdida de la extensibilidad y comienzo de los acortamientos marcados en la fibra muscular.

- Fase de post-rigor en que el músculo vuelve a hacerse extensible pero en menor magnitud que en la primera fase.

Dichas fases, según las investigaciones realizadas en el Instituto de Investigaciones de la carne de Langfootf, pueden variar considerablemente según lo hagan las circunstancias premortem y la temperatura y establece las siguientes variantes que resumimos a continuación:

- En animales bien alimentados e inmovilizados farmacológicamente antes de la muerte, con temperatura ambiente de $17^{\circ} \mathrm{C}$, y con temperatura ambiente de $37^{\circ} \mathrm{C}$. No es aplicable a nuestro caso.

- Animales agitados o que sufren convulsiones antes o durante la muerte: La duración del rigor es más limitada.

- Animales hambrientos o famélicos, sedados antes de la muerte: La duración total del rigor esta disminuida.

Estas variantes son debidas a la diferente cuantía de los depósitos de ATP, fosfocreatinina y glucógeno, así como también el tipo de $\mathrm{pH}$ inicial del rigor. Los músculos de los animales agitados muestran niveles de ATP, fosfocreatinina y glucógeno muy bajos así como el valor del pH que es bajo en razón de la cantidad de lactato originado durante la agitación premortem.

Bendall ha observado también que el trabajo muscular en el rigor mortis, aumenta con la temperatura y los valores altos de $\mathrm{pH}$. Se ha invocado la acidez muscular como factor desencadenante, pero si anulamos la acidez mediante una intoxicación por ácido monoyodoacético, la rigidez aparece igualmente. Hoy el rigor mortis se clasifica como rigor ácido, caracterizado por ser de gran duración y sincronismo con la temperatura, y rigor alcalino, con $\mathrm{pH}$ superior a 7,2 de rápida aparición, incluso a temperatura ambiente y que provoca un gran endurecimiento y acortamiento de la fibra muscular. Favorece su aparición el predominio de musculatura de tipo rojo y el estrés o lucha durante la agonía. Perfectamente aplicable al caso del Hombre sindónico.

Sí se ha comprobado la importancia de la hidratación muscular, que la retrasa, mientras que la deshidratación la acelera. El grado de hidratación es también un factor esencial. Por esta razón, cuando existe un anasarca, o edemas, el exceso de hidratación dificulta su aparición. Incluso puede aparecer con un ritmo distinto en los miembros en función de su contenido en agua. El fenómeno explica la rigidez de miembros superiores mayor que de los inferiores que se describe por los autores.

La deshidratación del músculo origina una rigidez inmediata, como han puesto de manifiesto Lacassagne y Martín inyectando deshidratantes químicos (cloroformo, cloruro cálcico) y comprobado por Küsmaul (agua de cal, potasa, vinagre, agua nitrada y carbonato potásico) y por Coze valiéndose del cloroformo. En estos casos la rigidez aparece rápidamente. Así ocurre en enfermedades deshidratantes (cólera, grandes hemorragias, caquexias, muerte por sed) y es posible conseguir experimentalmente diversos grados de rigidez inyectando regionalmente las masas musculares. Las hemorragias grandes actúan igualmente al disminuir enormemente la hidratación tisular y, entonces, la rigidez se hace precoz, débil y corta. Toda la Pasión de Jesús mantiene una deshidratación progresiva; el mismo hecho de la desnudez en la cruz facilita la evaporación del copioso sudor y la deshidratación acentuada.

Por tanto, a la vista de lo expuesto en relación a las causas modificadoras intrínsecas y extrínsecas que actúan sobre la rigidez, es coherente y racional que la rigidez se hubiera desencadenado en fase precoz. 


\section{Diagnóstico diferencial}

Existen una serie de cuadros de contractura muscular postmortem que pueden simular una rigidez cadavérica y que exigen, por lo tanto, su diferenciación, en función de los datos de que disponemos en relación a la muerte de Jesús:

En la literatura médica podrían ofrecer problemas de identificación los cuadros siguientes:

- En primer lugar la rigidez cataléptica, histérica, hipnótica o esquizofrénica que han sido motivo de diagnósticos equivocados de muerte debido a una exploración defectuosa hecha superficialmente. Un estudio clínico somero demuestra la vitalidad del sujeto y la inexistencia de otros fenómenos cadavéricos; forzada la rigidez, recupera inmediatamente su posición; la excitación eléctrica origina respuesta muscular. En la Síndone los datos lesionales y los signos cadavéricos son tantos y de tal naturaleza que hacen imposible mantener, desde el punto de vista de la pericia medicolegal, que el Hombre de la Síndone estuviese vivo.

- La rigidez por el calor postmortem es otra posibilidad que en este caso se excluye ante la inexistencia de signos corporales de quemadura. Es debida a la desnaturalización y precipitación de las proteínas musculares por el calor. Es una rigidez permanente que al ser forzada produce desgarros musculares. Por otro lado, la morfología general del cuadro completa el diagnóstico.

- La posible rigidez por el frío también puede excluirse dadas las características de la imagen, sus antecedentes y los datos históricos. Es debida a la congelación del cadáver. La presión de las masas musculares produce un crujido característico por la rotura de los cristales de hielo que se han originado a nivel intracelular. Producida la descongelación, reaparece la rigidez específicamente cadavérica.

- Otra hipótesis que ha llegado a plantearse en los estudios sobre rigidez cadavérica es la de una posible rigidez de descerebración. Se trata de una contractura generalizada hipertónica de la musculatura esquelética, con extensión rígida de las cuatro extremidades, en rotación interna las superiores, aduccion de las inferiores y tendencia al opistótonos de la nuca y del tronco. Se presenta en lesiones pedunculares, hemorragia cerebral masiva. Ejemplo típico entre nosotros es la del toro apuntillado. Es una rigidez instantánea con la lesión. También debe excluirse porque al constituir un cuadro espás- tico reflejo, es incapaz de conservar la postura que tenía el sujeto en el momento de la muerte.

- Rigidez de decorticación. Se origina en lesiones más altas del tronco del encéfalo, inmediatamente cercanas a los hemisferios o intrahemisféricas bilaterales. Entonces encontramos al sujeto con una extensión rígida de las piernas y flexión hipertónica de las extremidades superiores. Por idénticas razones puede excluirse también.

- Tétanos. Se puede presentar una espasticidad generalizada debida a la acción difusa de la toxina sobre el sistema nervioso con liberación de las motoneuronas periféricas, pero la contractura que origina es distinta a la que se ofrece en la Síndone.

- Espasmo cadavérico: Se trata de una rigidez especial, de carácter vital que aparece en el momento de la muerte fijando la posición del cadáver. Puede ser general o parcial, segmentario, de la fisonomía o de la actitud.

En estos casos, la rigidez se instala sin transición alguna, inmediatamente después de la postrera contracción muscular. Esta contracción se mantiene fija y sin modificaciones posturales. La propia rigidez que vendría a continuación no hace sino fijar y acentuar el espasmo. Etienne Martín define el espasmo cadavérico como la persistencia después de la muerte de una contracción determinada voluntariamente en vida y que se continúa en el cadáver. No existe, por tanto, un período previo de relajación.

Se origina de modo preferente en las muertes violentas por arma de fuego, lesiones del S.N., especialmente acompañadas de hemorragias cerebrales, y en las muertes violentas acompañadas de convulsiones, muertes por fulguración y cuadros asfícticos, especialmente por sumersión, tétanos, intoxicaciones estrícnicas, comprobadas por Sommer, Haen, Gentz, Küsmaul, Tourdes y tantos médicos legistas. Se trata de una contractura muscular antigravitatoria que por su naturaleza es fácilmente detectable. Arnaud, Périer, Chenu en las guerras de Italia y Crimea, Neudurfer, Brinton, en las guerras de sucesión; Rossbach en la guerra francoprusiana, descrita también en nuestra Guerra Civil y, en general, en todos los campos de batalla.

Es un fenómeno muy raro. Polson y Gee, en 20 años de ejercicio solo vieron dos casos. Schneider añade que la mayoría de los casos publicados en la literatura son de origen bélico o 
secundarios a una electrocución. Bauman ha comprobado cómo la mayoría de estos casos se origina cuando existen lesiones craneales, cerebrales o del tronco cerebral y lesiones torácicas con posible embolismo gaseoso.

Su mecanismo no es bien conocido. La mayor parte de los autores, siguiendo a Brown-Sequard, explican el espasmo por mecanismos nerviosos, aunque en estos casos no esté interesada directamente la región bulboprotuberancial. En ellos, las lesiones causales de la muerte determinarían una inhibición que liberaría la medula. Gracias a ella, el sistema muscular conservaría su tono vital y aún lo exageraría a veces, fijando el cuerpo en la actitud que tenía en el momento de la muerte. Para Magnus y Sherrington, para Laves y Prokop, sería una forma de la rigidez de descerebración; sin embargo, hemos señalado más arriba las diferencias que excluyen esta etiología. Para Pérez Argilés sería debida a una descarga de acetilcolina. Sea como fuere el mecanismo todavía no se conoce en profundidad, ahora bien, su existencia constituye una realidad incuestionable. Peiró propone como elemento diferenciador de la rigidez de una contractura vital, forzar mecánicamente la acción muscular. Si se trata de rigidez, el miembro permanece en la nueva posición y no ocurre en las contracturas vitales que tratan de volver a la postura inicial.

Delgado Roig pone como ejemplo de un espasmo generalizado la imagen del Cristo de la Expiación de la Cofradía Sevillana del Museo, atribuida a Marcos Cabrera. Adalberto Pazzini ha descrito el mismo fenómeno en numerosas representaciones de la crucifixión de Cristo del siglo XIII. En el campo médico, Le Bec, en 1925, Barbet, Hynek, y De Vicentiis relacionan el fenómeno con el esfuerzo y tetanización muscular que sufrió Cristo en la Cruz a los que hay que sumar toda la serie de patologías complementarias que agudizaban el cuadro.

Sin embargo, en el caso que nos ocupa existe un dato que permite excluirlo. Thoinot señaló que "existe una diferencia capital entre rigidez precoz y espasmo cadavérico, pues la primera supone previamente la relajación muscular, tanto más corta cuanto antes se quedan rígidos los músculos. En el espasmo cadavérico no existe, en cambio la relajación intermedia, sucediéndose inmediatamente la contracción muscular y la rigidez. De ahí resulta que la rigidez precoz, por rápida que la supongamos, no puede fijar el cadáver en una postura adoptada durante los últimos instantes de la vida cuando esta posición es contraria a las leyes de la gravedad. La relajación muscular interviene, en efecto, para destruir esta posición, la cual permanece fija, por el contrario en el espasmo cadavérico". Existe un testimonio presencial que nos permite excluir un posible espasmo cadavérico y nos lo proporciona Juan en su Evangelio (Jn, 19. 30), "e inclinando la cabeza, entregó el espíritu”. La inclinación cervical es secundaria a la relajación muscular. Existió, por tanto, un período previo de relajación antes de instaurarse la rigidez propiamente dicha.

\section{Cronotanatodiagnóstico}

Contamos para reconstruir la evolución temporal con un punto de referencia fija y otro aproximado. Es una referencia fija que cuando el cadáver se retiró de la cruz, estaba en plena rigidez y esta estaba plenamente establecida porque mantuvo la postura que tenía en la cruz a pesar de las manipulaciones a que fue sometido.

Sabemos, por los cálculos realizados sobre el Sudario de Oviedo que, tras la muerte, se mantuvo colgado el cuerpo entre tres cuartos de hora y una hora, por lo tanto la evolución de la rigidez debió ser muy rápida.

\section{¿Cuándo se produjo la imagen?}

Siendo esto así, la existencia del rigor mortis implantado y su valoración temporal puede orientarnos sobre el momento en que se produjo la imagen a partir del momento de la muerte que es otra de las grandes incógnitas de la Síndone.

Porter recoge en su libro, sin que cite procedencia ni razones, que Gilbert Lavois había sugerido que el cuerpo del crucificado había sido envuelto en la sábana durante un tiempo no superior a dos horas y media desde su muerte, frente a otros autores como Rodante, que calculaba este periodo en treinta y seis horas aplicando los cálculos estándar medicolegales, criterio que copia Benítez. Igartua, al no detectar signos putrefactivos marca un plazo de menos de tres o cuatro días, si bien señala que el cadáver no estuvo demasiado tiempo en el lienzo que lo envolvía; Manuela Corsini proporciona la cifra de unas treinta horas, sin llegar a las cuarenta, en función de las teorías vaporográficas sobre la formación de la imagen que entonces se daban por válidas. 
Por otro lado, la evolución de la rigidez tanto si es prolongada como si es corta sigue un desarrollo equivalente, por lo tanto, teniendo como referencia que es completa a los 60 minutos de la muerte, y valorando las cifras medias que contempla la literatura podemos trasladar a una precoz los valores correspondientes.

En efecto se calcula que el comienzo de una rigidez normal media estandar oscila entre 2 y 4 minutos. En el caso del Hombre de la Síndone el comienzo estaría entre 20 y 48 minutos; a la hora, la rigidez sería completa y máxima en un período comprendido entre 2 y 3 horas, siendo el período medio para el comienzo de la desaparición de 24 a 36 horas. En el caso que estudiamos el máximo posible para que el Hombre de la Síndone nos ofrezca una imagen de contractura estaría entre las 3 y 6 horas.

El momento en que se vence la rigidez de los brazos debe ser cuando se procede al traslado o a la somera limpieza y amortajamiento del cadáver. La rigidez vuelve a establecerse unas dos horas, aproximadamente, tras esa maniobra.

Teniendo en cuenta estas cifras y tomando como base los plazos y movimientos que sufrió el cadáver, que conocemos a través de los relatos evangélicos y, sobre todo, por el estudio del Sudario de Oviedo podríamos esquematizarlo del modo que se muestra en la Tabla 1.

\begin{tabular}{|c|c|c|c|c|}
\hline \multirow{2}{*}{\multicolumn{2}{|c|}{ HORARIO POSIBLE }} & \multicolumn{3}{|c|}{ RIGIDEZ CADAVÉRICA } \\
\hline & & \multirow{2}{*}{$\begin{array}{l}\text { Plazo } \\
\text { corto }\end{array}$} & \multirow{2}{*}{$\begin{array}{l}\text { Plazo } \\
\text { largo }\end{array}$} & \multirow{2}{*}{$\begin{array}{l}\text { Plazo } \\
\text { estándar }\end{array}$} \\
\hline Muerte & $15 \mathrm{~h}$ & & & \\
\hline Comienzo rigidez & $\begin{array}{l}15 \mathrm{~h} 20 \mathrm{~min} \text { a } \\
15 \mathrm{~h} 48 \mathrm{~min}\end{array}$ & $19,8 \min$ & $48 \mathrm{~min}$ & $2-4 h$ \\
\hline $\begin{array}{l}\text { Se descuelga. } \\
\text { Rigidez completa }\end{array}$ & $16 \mathrm{~h}$ & $12 \mathrm{~h}$ & $12 \mathrm{~h}$ & \\
\hline Entrega del cadáver & $17 \mathrm{~h}$ & & & \\
\hline Posible rigidez Máxima & & $2 \mathrm{~h} 15 \mathrm{~min}$ & & $24 \mathrm{~h}$ \\
\hline Traslado y mortaja & $17 \mathrm{~h} 30 \mathrm{~min}$ & & & \\
\hline $\begin{array}{l}\text { Se vence rigidez } \\
\text { de los hombros }\end{array}$ & 17 h 30 min & & & \\
\hline Posible rigidez Máxima & & & $4 \mathrm{~h}$ & $24 \mathrm{~h}$ \\
\hline $\begin{array}{l}\text { Restablecimiento de la } \\
\text { rigidez de los brazos }\end{array}$ & $19 \mathrm{~h} 30 \mathrm{~min}$ & $3 \mathrm{~h}$ & $6 \mathrm{~h}$ & \\
\hline $\begin{array}{l}\text { Comienza } \\
\text { desaparición rigidez }\end{array}$ & $21 \mathrm{~h}$ & & & $36-48 \mathrm{~h}$ \\
\hline
\end{tabular}

Por lo tanto el plazo para que se produzca la imagen del Hombre de la Síndone en estado de rigidez total debe estar comprendido entre las 19,30 (mínimo) y las 21 horas (máximo) desde el momento de la muerte.

\section{Consideraciones finales}

Las cifras a que hemos Ilegado son, evidentemente aproximadas, y precisan su contraste y discusión por otros especialistas en Medicina Legal y Forense, pero muy probables a la vista de los conocimientos que existen actualmente sobre la rigidez cadavérica y su evolución y la posible influencia de las circunstancias modificadores.

Evidentemente estas cifras nos plantean nuevos problemas para estudiar y que trascienden a la medicina pero que el estudio medicolegal plantea.

\section{Agradecimientos}

Mi gratitud a Guillermo Heras, que con sus opiniones y nuestras conversaciones sentó las bases para esta comunicación, a María Teresa, mi mujer, que, como siempre, ha tenido la paciencia de corregir críticamente este texto.

\section{Bibliografía}

- Abenza Rojo JM. Rigidez Cadavérica. Tesina de Licenciatura. Facultad de Medicina. Universidad Complutense. Madrid. 1989.

- Arnaud.- De l'attitude des morts sur les champs de bataille. Recuil des memoires de medicine militaire. 1880. Cit. Royo-Villanova.

- Alarcón J. El Quinto Evangelio. Ed. Alonso. Madrid, 1984. Pág. 156.

- Anson F. Después del carbono 14. Arcaduz. Ed. Palabra. Madrid, 1989. Pág. 99.

- Anson F. La Sábana Santa. Últimos hallazgos. Arcaduz Ed. Palabra. Madrid, 1994.

- Aso J, Corrons J, Cobo JA. El intervalo postmortal. Masson. Barcelona 1998. Pág. 31-32, 34-5.

- Baima Bollone PL. L'Uomo della Sindone era cadavere. Sindon. VI, 7: 39-47, 1994.

- Baima Bollone PL. Sindone: La prova. Mondadori. Milano, 1998. Pág. 77 y ss.

- Balthazard V. Manual de Medicina Legal. Salvat Ed. Barcelona, 1926. 
- Balthazard V. Medicina Legal. Salvat Ed. Barcelona, 1933. Pág. 567-9.

- Barahona I. Lecciones De Medicina Legal. TPI. Marcelino Rodríguez. Salamanca, 1908. Págs. 379-84.

- Barbet P. La Pasión de N .S. Jesús-Christ selon le Chirurgien. Dilen \& Cia. Ed. Issoudun (Indre), 1950. Pág.96-7.

- Barbet P. La Pasión du Christ selon le Chirurgien. Apostolat des Editions. Paris, 1965. Pág. 193.

- Bate-Smith EC \& Bendall JR. Factor determining the time course of rigor mortis. J. Phisiol 1949;110:47-65.

- Baumann J. Ueber kataleptische Totenstarre. Dtsch. Z. ges. Gerichtl. Med. 1923:2: 647-70.

- Bendal JR. Postmortem changes in muscle. In The structure and function of muscle. V. II. Structure. 2. Academia Press. 1973. Págs. 344-309.

- Bendal JR. The structure ND Function of Muscle. Borne. Academic Press. Vol 3, 1961 Pág. 364.

- Bendal JR. Muscle molecules and movements. Heinemann. London, 1969, Pág 206.

- Bendal JR. Postmortem changes in muscle. En Bourne Ed.- The structure and function of Muscle. Academia Press. N. Cork, London. Vol. II. 1973. Pag 244-306.

- Benítez JJ. El enviado. Plaza \& Janés. Barcelona, 1994.

- Bonnet EFP. Medicina Legal. Vol. I. López Libreros, Buenos Aires, 1980. Págs. 296-8.

- Biggio P. Cause di morte dell'uomo della Sindone. Sindone II, 2: 33-8, 1990.

- Briand J, Bouis J, Casper JL. Manual completo de Medicina Legal y Toxicología. Vol.II. Moya y Plaza, Madrid, 1873. Págs. 353-5.

- Brown-Sequard. Proc. R. S. mayo, 1861. Cit. Taylor, Pág. 54

- Bucklin R. The Shroud of Turin: A Pathologist's viewpoint. Legal Medicine Annual. 1982.

- Bucklin R. The medical aspects of the crucifixion of Christ. Sindon III, 7: 7-11, 1961.

- Bucklin R. "An autopsy on the Man of the Shroud". Actes du III Symposium scientifique international. Ed. du Ciel. Nice, 1997. Pág. 99.

- Cairns F. Med. J. Austral. 2: 585, 1952.

- Caminal S. "Estudio de la crucifixión según la Sábana Santa". En Huidobro L. El Misterio del Santo Sudario. Biblioteca Sindoniana. Barcelona, 1957. Págs. 56-7.
- Camps L. Gradwohl's Legal Medicine. John Wright \& Son. London, 1971.

- Cappi M. La Sindone dalla A alla Z. Ed. Messagero. Padova, 1997. Pág. 65.

- Casas JD, Rodríguez Albarrán MS. Manual de Medicina Legal y Forense. Colex, Madrid, 2000. Págs. 1179-84.

- Conway D, Sakai t. Caffeina contracture. Proc. Nat. Acad. Sci (USA), 46: 897, 1960.

- Corin G. Los fenómenos cadavéricos. Los Progresos de la Clínica. 4: 305, 1914.

- Corsini de Ordeig M. El Sudario de Cristo. Rialp. Madrid, 1976. Pág. 206.

- Dix J, Graham M. Time of death, decomposition and identification. CRC Press. Boca Raton, London, New York, Washington, D.C. 1999. Págs. 2-4.

- Delgado Roig J. Los signos de muerte en los crucificados de Sevilla. Edelce. Sevilla, 1951.

- Derobert L. Médicine Légale. Flammarion. París, 1974.

- Derobert L. Eléments de Médicine Légale. Ed. Medicals et Universitaires. Paris, 1977.

- Dolinak D, Matshes E, Lew E. Forensic Pathology. Elsevier Academic Press. Burlington, San Diego, London, 2005. Págs. 532-3.

- Domínguez JJ. "La Síndone. Estudio médico". En La Síndone de Turín. Estudios y Aportaciones. Centro Español de Sindonologia. Valencia, 1997. Págs. 110-1.

- Hessge CL, Madea B. Methodes zur Bestimmung der Todeszeit an Leichen. Schmidt - Römhild. Lübeck, 1988.

- Husband HA. The Student's Hand-Book of Forensic Medicine and Medical Police. Livingstone. Edimburg, 1877.

- Evans WED. The Chemistry of death. Charles Thomas. New York, 1963.

- Favero F. Medicina Legal. Vol. II. Martins. Sao Paulo, 1973. Págs. 99-113.

- Franchini A. Medicine Legale. CEDAM. Padova, 1985.

- Gisbert JA, Villanueva E, Gisbert M. Fenómenos cadavéricos. En Villanueva E (Ed.). Gisbert Calabuig. Medicina Legal y Toxicología. Masson, Barcelona, 2004. Pags. 195-9.

- Gonzales TA, et al. Legal Medicine, Pathology and Toxicology. Appleton-Crofts-Cantury. New York, 1954.

- Gordon I, Shapiro HA. Forensic Medicine. Churchill Livingstone. Edinburg, 1975. 
- Hamilton-Paterson JL, Johnson EWM. Post-mortem glycolisis. J Pathol Bacteriol 1940;50:473-82.

- Hynek RW. El aspecto físico de Jesús en el Santo Sudario. Bibliotheca Sindoniana. Barcelona, 1954. Pág. 77.

- Hynek RW. Lo que revela el Santo Sudario a un convertido. Bibliotheca Sindoniana, $\mathrm{n}^{\circ} 10$. Barcelona, 1951. Pág. 65.

- Igartua JM. El enigma de la Sábana Santa. Ed. Mensajero. Bilbao, 1988. Págs. 72,77,80.

- Igartua JM. La Sabana Santa es auténtica. Ed. Mensajero. Bilbao, 1990. Pág. 174.

- Izquierdo Asensi A, Catalá Catalá A. Rigidez cadavérica: etiología e importancia en el cronotanatodiagnóstico. Medicamenta. Madrid, julio, 1949.

- Judica-Cordiglia G. L'uomo della Sindone e'il Gesu dei Vangeli?. Ed. Fundazione Pelizzia, Chiari (Brescia), 1974.

- Knight B. The estimation of the time since death in the early postmortem period. Edward Arnold. London, 1995.

- Knight B. Forensic Pathology. Edward Arnold. London, 1996.

- Krompecher T. Rigor mortis: estimation of the time since death by evaluation of cadaveric rigidity. En Henssge $\mathrm{C}$ et al. The estimation of the time since death in the early postmortem period. Arnold. London, 2002. Págs. 144-60.

- Krompecher T et al. Experimental evaluation of rigor mortis. VI. Effects of anti and post-mortem electrocution on the evaluation of rigor mortis. Forensic Sci Int 1988;38:27-35

- Kusmault A. Ueber die Todtenstarre und die ihr nahe verwandten Zustände von Muskelstarre, mkit besonderer Berücksichtigung auf die Staatsarzneikunde. Vierteljahrsschr. prakt. Heil (Prag) 1e: 67-115, 1888. Cit Krompecher.

- La Sindone.- Cominato per l'ostensione. S.d. Pag 12.

- Lacassagne A. Compendio De Medicina Legal. Herederos de Juan Gili. Barcelona, 1912. Vol. I. Págs. 608-13.

- Laves W. Ueber die Totenstarre. Dtsch. Z. Ges. Gerichtl Med 1948-49;39:186-98.

- Lecha-Marzo A. Tratado de Autopsias y Embalsamamientos. Los Progresos de la Clínica. Madrid, 1911. Págs. 12-4.

- Löring J. La Sábana Santa dos mil años después. Planeta testimonio Planeta. Barcelona, 2000. Págs. 101 y 149
- Louis. Obras diversas de cirugía, cuarta carta, de la certeza de los signos de muerte. Pág. 119. Cit. Orfila. Vol. I. Pág. 453.

- Lyle H, Stemmer K, Cleveland F. J Forensic Sci 1959;4:167.

- Mallach HJ. Zur frage der Todeszeitbestimmung. Berl Med 1964;18:577-82.

- Mallach HJ, Mittmeyer HJ. Totenstarre und Totenflecke. Z Reichtmed 1969;69:70-8.

- Marañón G, Balcells A. Manual de Diagnóstico Etiológico. Espasa-Calpe. Madrid, 1984. Págs. 797, 822, 823, 824.

- Macaggi D, Manunza P, Franchini A. Medicina Legale. Idelson. Napoli, 1957.

- Martin E. Manual de Medicina Legal. Salvat Ed. Barcelona, 1942. Págs. 178-82.

- Martin E, Mazel P. Contribution a l'étude du spasme cadaverique. Ann Med Leg Crim Pol Scient. Enero 1922.

- Marvizon J. La Sábana Santa. Ed. Giralda, S.L. Sevilla 1998. Págs. 59-60.

- Mende. Ansfuchrliches Handbuch der Gerichtliche Medizin. Vol. III. Pág. 405. Cit. Orfila. Pág. 453.

- Mueller B. Geritliche Medizin. Springer. Berlin, 1953.

- Muñoz-Tuero LM, Ladrón de Guevara LM, Ladrón de Guevara J, Villalaín Blanco JD. Técnicas de la autopsia judicial. Nociones fundamentales. Escuela de Medicina Legal, Madrid, 1968, 57 págs.

- Muñoz-Tuero LM, Villalaín Blanco JD. Manual de autopsias de la armada norteamericana. Traducción. Publicaciones del Ejército, de la Marina y de las Fuerzas Armadas. Madrid, julio de 1960.

- Muñoz-Tuero L M. Tanatología. Escuela de Medicina Legal. Madrid, 1975. Págs. 61-3, 82.

- Mueller B. Gerichtlichte Medizin. Springer Verlag. Berlin, 1953.

- Niderkorn PF. Cit. Polson.

- Novelli G. La Sindone un caso ancora aperto. Supl L'Eco di S Gabrielle 1993;6:18.

- Oliveira de Sa FM. Cronotanatodiagnosis. Tesis doctoral. Universidad de Coimbra. Coimbra, 1966.

- Orfila M. Tratado de Medicina Legal. Imprenta de D. José María Alonso. Madrid, 1847. Págs. 453-8.

- Palmieri A. La anatomía de los crucifijos de los siglos XII y XIII y una hipótesis fisiopatológica. Gaceta Sanitaria. Barcelona, núm. 1, enero-febrero 1951. 
- Palmieri A. Medicina Forense. Morano. Napoli, 1964.

- Peiró PM, Rodrigo J. Elementos de Medicina y Cirujía legal arreglados a la legislación Española. Agustín Sevil. Zaragoza, 1832. Págs. 167-8.

- Payne James J, Busuttil A, Smock W. Forensic Medicine. GMM. San Francisco, London, 2003. Págs. 100-1.

- Piga A. Medicina Legal de Urgencia. Ed. Mercurio. Madrid, 1928. Págs. 163-5.

- Polson CJ. The Essentials of Forensic Medicine. Pergamon Press. Oxford, 1962.

- Polson CJ, Gee DJ. The essentials of Forensic Medicine. Pergamon Press. Oxford, 1973.

- Ponsold A. Lehrbuch der geritchlichen Medizin. Georg Thieme. Stuttgart. 1957.

- Porter C. La Sábana Santa. EDAF. Madrid, 2005. Pág. 98.

- Prokop O. Die Totenstarre (Rigor mortis). En Prokop O, Gohla W Eds. Forensische Medizin. Fischer Verlag. Stuttgart, 1976.

- Prokop O. Supravital Erscheinungen. En Prokop O, Gohla W. Eds. Forensische Medizin.Volk und Gesundheit Verlag 1975. Págs. 16-27.

- Rodríguez Pazos M. Ultraestructura de los cambios postmortem en el músculo estriado. Tesis doctoral. Barcelona, 1976.

- Rojas N. Medicina Legal. El Ateneo. Buenos Aires, 1976. Págs. 131-2.

- Royo-Villanova R. El proceso bioquímico de la rigidez cadavérica. Gaceta Médica Española 1958;XXXII(1):37.

- Royo-Villanova R. Rigor Mortis. Ed. Lex. Madrid, 1960.

- Saphiro H. J Forensic Med 1954;1:144.

- Saukko P, Knight B. Knigtht's Forensic Pathology. Arnold. London, 2004. Págs. 60-3.

- Schleyer F. Leichenveränderungen. Todeszeitbestimmung im früh-posmortalen Intervall. En Muller B Ed. Gerichtliche Medizin, Bd. 1. Springer Verlag. Berlin, 1975.

- Schleyer F. Determination of the time of death in the early postmortem interval. In Curry AS. Methods of
Forensic Science. Vol. II. John Wiley. London. 1963. Págs. 253-90.

- Sherrington CS. The integrative Action of the Nervous System. A. Constable and Co. London, 1908.

- Siliato MG. El Hombre de la Sábana Santa. BAC Popular. Madrid, 1987. Págs. 210-1, 217, Apéndice.

- Siliato MG. La Sábana Santa. PPC. Madrid, 1998. Págs. 249, 251.

- Simpson K. Medicina Forense. Espasa, S.A. BarceIona, 1981. Págs. 25-6.

- Sole M. La Sábana Santa de Turín. Mensajero. Bilbao, 1985. Págs. 262- 264, 374-6.

- Spitz WU \& Fisher RS. Medicolegal Investigation of death. Charles C. Thomas Publ. Springfield. III. 1980. Págs. 14-6.

- Stevenson KE, Habermans GR. Dictamen sobre la Sábana de Cristo. Planeta. Barcelona, 1988. Págs. 16-185.

- Taylor AS. Tratado de Medicina Legal. Tipografía Enrique Teodoro. Madrid, 1890. Págs. 53-5.

- Teke A. Medicina Legal. Mediterráneo. Santiago de Chile, 2001. Págs. 188-90.

- Thoinot L. Tratado de Medicina Legal. P. Salvat. Barcelona, 1923. Vol. I. Págs. 75-95.

- Valdés-Ruíz M. El milagro de la Sabana Santa. Manuel Valdés Ruiz. Valencia, 1988. Pág. 297.

- Villalaín JD. Policía Científica. Autor, Madrid, 1981, 2 vol. de 467 y 631 pags.

- Villalaín JD. Asfixias Medicolegales. Apuntes Elaborados para el II Curso de Especialización en Inspecciones Oculares. Academia Superior de la Policía. Avila, 1987.

- Villalaín JD. Momento en que se produjo la imagen sindónica. Valoración de la Medicina Legal. Convención CES 2006. Universidad Católica de Valencia "San Vicente Mártir". Sede de Santa Ursula. 1 de mayo de 2006.

- Vock R, Hein PM, Metter D. Tod im tetanische. Anfall Z Rechtsmed 1984;92:231-7. 